\title{
Numerical Comparison of Optimal Charging Schemes for Electric Vehicles
}

You, Shi; Hu, Junjie; Pedersen, Anders Bro; Andersen, Peter Bach; Rasmussen, Claus Nygaard; Cha, Seung-Tae

Published in:

Proceedings of IEEE PES General Meeting

Link to article, DOI:

10.1109/PESGM.2012.6345356

Publication date:

2012

Link back to DTU Orbit

Citation (APA):

You, S., Hu, J., Pedersen, A. B., Andersen, P. B., Rasmussen, C. N., \& Cha, S-T. (2012). Numerical Comparison of Optimal Charging Schemes for Electric Vehicles. In Proceedings of IEEE PES General Meeting IEEE. https://doi.org/10.1109/PESGM.2012.6345356

\section{General rights}

Copyright and moral rights for the publications made accessible in the public portal are retained by the authors and/or other copyright owners and it is a condition of accessing publications that users recognise and abide by the legal requirements associated with these rights.

- Users may download and print one copy of any publication from the public portal for the purpose of private study or research.

- You may not further distribute the material or use it for any profit-making activity or commercial gain

- You may freely distribute the URL identifying the publication in the public portal

If you believe that this document breaches copyright please contact us providing details, and we will remove access to the work immediately and investigate your claim. 


\title{
Numerical Comparison of Optimal Charging Schemes for Electric Vehicles
}

\author{
S. You, Member, IEEE, J. Hu, Student, IEEE, A.B. Pedersen, Student, IEEE, \\ P.B. Andersen, Student, IEEE, C. N. Rasmussen and S. Cha
}

\begin{abstract}
The optimal charging schemes for Electric vehicles (EV) generally differ from each other in the choice of charging periods and the possibility of performing vehicle-to-grid (V2G), and have different impacts on EV economics. Regarding these variations, this paper presents a numerical comparison of four different charging schemes, namely night charging, night charging with V2G, 24 hour charging and 24 hour charging with V2G, on the basis of real driving data and electricity price of Denmark in 2003. For all schemes, optimal charging plans with 5 minute resolution are derived through the solving of a mixed integer programming problem which aims to minimize the charging cost and meanwhile takes into account the users' driving needs and the practical limitations of the EV battery. In the post processing stage, the rainflow counting algorithm is implemented to assess the lifetime usage of a lithium-ion EV battery for the four charging schemes. The night charging scheme is found to be the cheapest solution after conducting an annual cost comparison.
\end{abstract}

Index Terms-Electric vehicle, mixed integer programming, optimal charging, rainflow counting, vehicle-to-grid, V2G

\section{INTRODUCTION}

$\mathrm{T}$ HE technique of optimal charging in the context of deregulated electricity markets, sometimes referred as smart charging, has recently caught a lot of attention, as there is a tremendously growing need for electrifying the transportation sector [1]. The term "optimal” can be generally interpreted from two perspectives: the EV owners and the power system operators.

From the EV owners' point of view, "optimal” can be simply interpreted as minimizing the cost of charging while guaranteeing their need for driving. In [2]-[5], this perspective has been intensively investigated based on various modeling techniques including linear programming, dynamic programming and quadratic programming. A common impression inferred by these studies is that the optimal charging as a feasibility solution can considerably reduce the cost of charging; however, to support a large-scale roll-out of $\mathrm{EV}$, services like V2G have to be offered by EV or EV fleet to improve the EV economy [6]-[7].

From the power system operators' point of view, "optimal" can be generally interpreted as a complex objective which aims to maximize the advantages of $\mathrm{EV}$ and minimizes its disadvantages. The energy storage nature of $\mathrm{EV}$ makes it a potential solution to many power system problems, such as load shifting and frequency regulation. Meanwhile, its nature of being a mobile electrical load challenges the power system operation, as an inappropriate integration could easily cause voltage issues and overloading in the distribution network. Regarding this aspect, intensive studies have been done in [8][11], wherein coordinated charging schemes for an EV fleet with either centralized or decentralized control structures are developed to handle grid constraints and meet the driving requirements at the same time.

Apart from the different perspectives of the two groups of stakeholders, the optimal charging schemes are also heavily dependent on a large number of factors, including:

1) range of uncertainty related to electricity price and driving pattern [12];

2) fidelity of the EV battery models which varies from linear to non-linear;

3) modeling approaches used to describe the charging process and the associated optimization techniques which span from the conventional linear programming to the genetic algorithms [13];

4) time resolution used in the various simulations that spans from a few minutes up to hours; and so on.

In this study, four different optimal charging schemes for EV, namely night charging, night charging with V2G, 24 hour charging and 24 hour charging with V2G, are formulated as a set of mixed integer programming problems. Based on the practical driving data and electricity prices collected for Denmark in 2003, these charging schemes are simulated at a time resolution of 5 minute. Objectives for the four charging schemes are set to charging cost minimization, due to the fact that today's EV penetration is relatively low compared to the other Distributed Energy Resources (DER) technologies and some distribution grids can handle the penetration level up to $20 \%$ [14]. In the following text, the mathematical formulation for various optimal charging schemes is described in Section II. Section III presents the numerical model development. Results and discussions are summarized in Section IV, wherein battery life and annual cost comparison among the four schemes are included. Section V concludes the study.

\section{PROBlem Formulation}

This work is part of project 'EcoGrid EU' with EU funding.
The authors are all with the Centre for Electric Technology, Department of Electrical Engineering, Technical University of Denmark, Kgs. Lyngby DK2880, Denmark (e-mail:\{sy, junhu, abp, pba, cnras, stc\} @elektro.dtu.dk).
Today, energy procurement of EVs into the power 
system is very market-driven, given the fact that the economy of EVs can be improved by market participation via appropriate aggregation services, e.g. Virtual Power Plants (VPP). A comprehensive analysis of different aggregation setups has been carried out by the Danish EDISON project in the last few years [15]. The report from the EDISON project suggests three methods that can facilitate the EVs' participation in today's electricity spot market:

1) The retailer broadcasts the electricity price once a day to the individual $\mathrm{EV}$ owners and the $\mathrm{EV}$ owners therefore make appropriate charging schedules according to the known price and the local intelligence;

2) The charging strategy can be a simple time of day charging based on EV owners' empirical knowledge on when the electricity price is relatively cheap, such as at night;

3) THE charging of EVs can be scheduled or controlled by a fleet operator based on a contractual setup, where the central intelligence is more relied upon.

In this study, the first integration method is utilized and modified to accommodate four different charging schemes:

1) Night charging: the charging period is constrained to be from 7pm to 7am, and discharging is not allowed;

2) Night charging with V2G: the charging or discharging actions can be performed between $7 \mathrm{pm}$ to $7 \mathrm{am}$;

3) 24 hour charging: the charging can be performed anytime when the $\mathrm{EV}$ is not in use, and discharging is not allowed;

4) 24 hour charging with V2G: the charging or discharging actions can be performed anytime in a day.

Since the market segment of today's EVs is primarily urban area due to the battery capacity limitation and public health concerns, this study firstly assumes that the charging infrastructure is available everywhere in the studied urban area, indicating that the EVs can be charged or discharged as long as they are not used for driving. Further, the terminology "V2G" used in this study refers to selling the battery energy back to the grid on an hourly basis at prices set the previous day; while in other literature V2G is normally referred to a mechanism that activates the provision of ancillary services.

To enable the comparison study, a mixed integer programming formulation is developed and solved using Matlab to find the optimal solution for each charging scheme applied to an individual EV. The common objective of each charging scheme, as in (1), is to minimize the cost of charging given the broadcasted electricity price $Q(i)$ and energy required for driving $E_{d}(i)$; meanwhile, the four charging schemes are enabled separately by turning on/off the binary variables $u_{1}(i)$ and $u_{2}(i)$.

$$
\min \sum_{i=1}^{N}\left\{\Delta E_{c}(i) \cdot Q(i) \cdot \frac{u_{1}(i)}{\eta_{c}}+\Delta E_{d}(i) \cdot Q(i) \cdot u_{2}(i) \cdot \eta_{d}\right\}
$$

$$
\left\{\begin{array}{l}
E(i)=E_{0}+\sum_{k=1}^{k=i}\left\{\Delta E_{c}(k) \cdot u_{1}(k)+\Delta E_{d}(k) \cdot u_{2}(k)-E_{d}(k) \cdot u_{3}(k)\right\} \\
\delta_{\min } \cdot E_{\text {nom }} \leq E(i) \leq \delta_{\max } \cdot E_{\text {nom }} \\
E_{d}(i+1) \cdot u_{3}(i+1) \leq E(i) \\
0 \leq \Delta E_{c}(i) \leq P_{c, \max } \cdot \eta_{c} \cdot \Delta t \\
-\frac{P_{d, \max }}{\eta_{d}} \cdot \Delta t \leq \Delta E_{d}(i) \leq 0 \\
u_{1}(i)+u_{2}(i)+u_{3}(i)=1
\end{array}\right.
$$

where the planning duration is divided into $N$ time intervals with $i$ denotes the number of sequence and $\Delta t$ denotes the time length of each interval. Decision variables $\Delta E_{c}(i)$ and $\Delta E_{d}(i)$ represent the energy charged into and discharged from the battery in each time interval respectively, while the other three binary variables $u_{1}(i), u_{2}(i)$, and $u_{3}(i)$ indicate the on/off status of charging, V2G and driving for each corresponding time interval. To facilitate the expression, an intermediate variable $E(i)$ is introduced to represent the energy level of the battery in the end of each time interval.

Parameters $E_{\text {nom }}$ and $E_{0}$ represent the nominal energy capacity and the initial energy of the battery in the planning period, while the charging and discharging efficiency are represented by $\eta_{c}$ and $\eta_{d}$. The maximum power exchanged between the EV inverter and the electrical grid is expressed by $P_{c, \text { max }}$ and $P_{d, \text { max }}$ respectively during charging and discharging processes, which constrains the maximum energy exchanged between the EV and the grid. For battery life concerns, $\delta_{\min }$ and $\delta_{\max }$ are further introduced to represent the manufacturer recommended sate of charge (SOC) range.

Explanations for the inequality constraints can be found in [3] wherein a similar problem is described using linear optimization by the same group of authors. Compared to the previous study, a major improvement is made in this study by formulating various charging schemes in a more generic and flexible way. Power performance of the battery is not included in this paper; however, given the energy performance within a certain time, the power performance can be derived simply by elaborating on the charging schemes e.g., constant power and constant current.

\section{NuMERICAL MODEL DEVELOPMENT}

For this numerical case study, the optimal charging plans for different charging schemes are derived for the next day with 5 minute resolution given the broadcasted electricity price and EV owners pre-defined driving requirement. The assumption of flawless forecasting made in this paper aims to resemble the best case scenario for different charging schemes. In practice, EV owners may not be able to precisely forecast their driving needs for the next day with 5 minute resolution in practice, which could result in higher cost for charging.

The daily optimization is further repeated over the course of a month to retrieve a more general charging performance. In this section, the selected battery model parameters, the driving information and the source of electricity hourly prices are briefly explained.

s. t. 


\section{A. Battery Model}

Due to its high density on power and energy, the Lithiumion battery technology has been commonly adopted by the automotive industry for vehicle electrification. In this study, a $28 \mathrm{kWh}$ Lithium-ion battery is emulated to represent the battery of a medium size family car in Denmark. The SOC range is set to between $10 \%$ and $90 \%$, and the efficiency of charging and discharging are both assumed as $90 \%$. When the $\mathrm{EV}$ is grid connected, the maximum power of charging and discharging $P_{c, \text { max }}$ and $P_{d, \text { max }}$ are both set to $4 \mathrm{~kW}$, meaning the maximum power drawn from grid is $4.4 \mathrm{~kW}$ during charging and the maximum power received by grid is $3.6 \mathrm{~kW}$ during discharging. When the EV is used for driving, the maximum power discharged to drive the motor is not constrained by $P_{d, \max }$ as the discharging power is dependent on the driving needs e.g., acceleration, and can therefore be much larger than $4 \mathrm{~kW}$. For every single day, the initial SOC at 00:00 is assumed to be $50 \%$ to guarantee the need for very early morning driving. This assumption also indicates the energy exchanged between the battery and the grid (disregarding the round trip efficiency) within a day is equal to the energy used for driving in that day.

For the emulated Lithium-ion battery, a general relationship between the lifecycle and the Depth of Discharge (DOD) is illustrated in Fig.1.

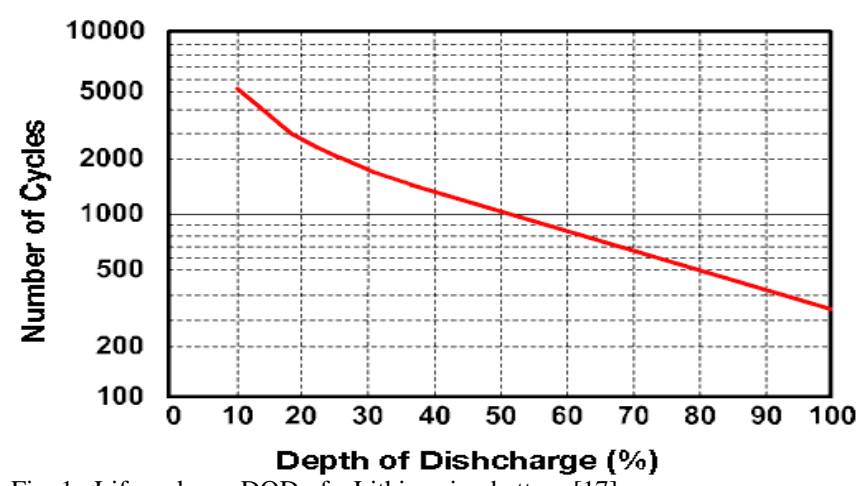

Fig. 1. Lifecycle vs. DOD of a Lithium-ion battery [17]

This relationship is approximately expressed by an exponential equation, as in (3), to support the later study on battery lifetime estimation.

$$
y=4515.2 \cdot e^{-0.0283 x}
$$

where $x$ is DOD in \%, $y$ is the number of expected cycles corresponding to any given value of $x$.

\section{B. Driving Information}

As few electric vehicles are already on the road, to support the related studies of EVs, a general assumption is taken in the way that vehicle electrification would have little impact on vehicle owners' driving pattern. In this study, driving information is taken from the 2003 AKTA survey[16], in which 360 cars in Copenhagen were tracked using GPS from 14 to 100 days. Due to the data incompleteness, the full-month record of the vehicle no. 32139 for March 2003 is picked out to support this study. The original data format is illustrated in Table I. Following the EV mileage assumption of $11 \mathrm{kWh} / 100 \mathrm{~km}$, the AKTA data is converted to energy required for driving with 5 minute resolution as shown in Fig.2. The frequent driving behaviors within a day are due to the fact that the selected vehicle is owned by a medium size family and shared by its family members.

TABLE I

SAMPLE OF DRIVING DATA FROM 2003 AKTA

\begin{tabular}{c|c|c|c}
\hline Start & Finish & Duration & Distance(m) \\
\hline $2003-3-2$ 9:31:57 & $9: 40: 23$ & $0: 08: 26$ & 21278 \\
\hline $2003-3-2$ 9:44:21 & $9: 46: 59$ & $0: 02: 38$ & 1685 \\
\hline $2003-3-210: 45: 38$ & $10: 50: 53$ & $0: 05: 15$ & 2855 \\
\hline $2003-3-210: 55: 56$ & $11: 00: 14$ & $0: 04: 18$ & 2336 \\
\hline $2003-3-212: 58: 36$ & $13: 02: 24$ & $0: 03: 48$ & 2206 \\
\hline $2003-3-215: 24: 21$ & $15: 27: 34$ & $0: 03: 13$ & 2110 \\
\hline $2003-3-217: 23: 21$ & $17: 26: 09$ & $0: 02: 48$ & 1658 \\
\hline $2003-3-218: 41: 00$ & $18: 44: 57$ & $0: 03: 57$ & 2587 \\
\hline $2003-3-218: 48: 39$ & $18: 52: 54$ & $0: 04: 15$ & 2689 \\
\hline
\end{tabular}

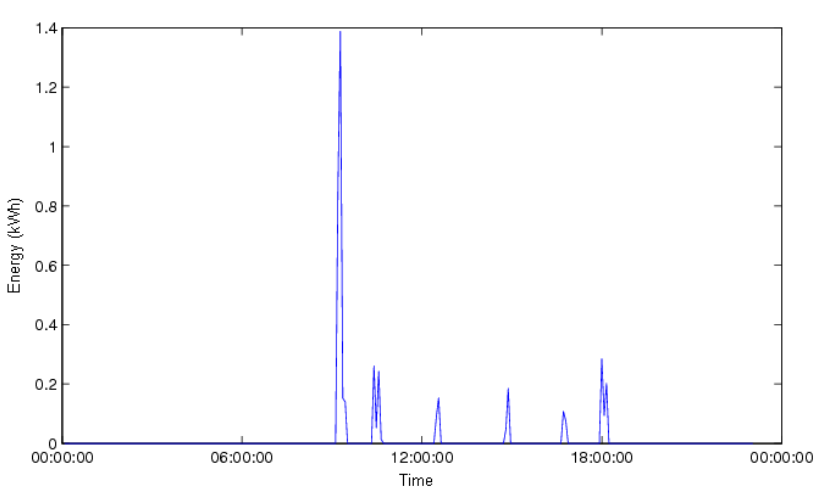

Fig. 2. Energy required for driving with 5 minute resolution on March $2^{\text {nd }}$

\section{Electricity Price}

The information of hourly electricity spot price of the Nordic power market is published and recorded on a daily basis by the market operator Nord Pool Spot. To guarantee the time consistency between electricity price and driving information, the electricity price of DK-west in March 2003, as illustrated by the figure on the left in Fig.3, is used for this study. The figure on the right in Fig. 3 depicts the hourly electricity price on March $2^{\text {nd }}$, which is used in the later case study.

With its average wind power production covering more than $25 \%$ of its annual electricity demand, the region DK-west is known as a good representation of a future energy system with large-scale stochastic renewable resources. In such a context, the volatility of the electricity price in DK-west is also relatively high, which to a great extent benefits the V2G operation. For other regions with much less price volatility such as DK-east, the V2G operation could hardly incur extra benefits due to the round-trip energy loss unless it is traded as ancillary services which is not considered in this study. 

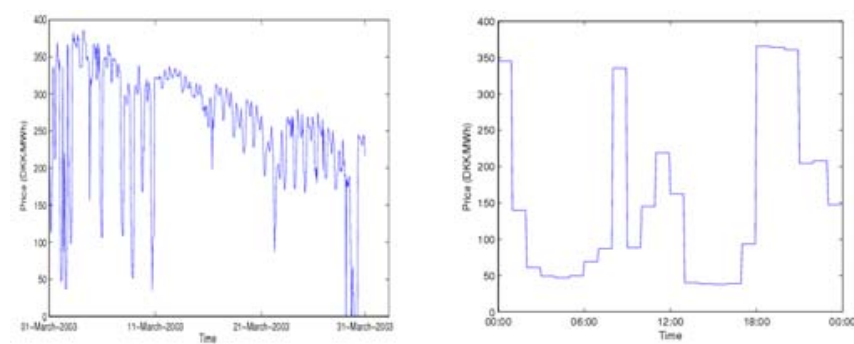

Fig. 3. Hourly electricity price of DK-west in the month of March 2003 (to the left), and on the day March $2^{\text {nd }}$ (to the right)

\section{RESUlts AND DisCUSSIONS}

In this section, the intra-day and the monthly charging behavior of the four different charging schemes are illustrated, and the short term economic performance, the lifetime consumption as well as the annualized cost for each charging scheme are calculated and compared.

\section{A. Intra-day Performance}

The result of an intra-day study for March $2^{\text {nd }}$, is illustrated by Fig. 4, where the blue line represents the energy requirement for driving and the red lines represents the energy charged (positive)/discharged (negative) for different charging schemes. Among the charging profiles, it can easily be found that the hours with cheap electricity are mostly selected for all schemes; however as the lowest electricity price occurs around $3 \mathrm{pm}$, for the 24 hour charging scheme, the charging is performed in that period instead of midnight. For charging schemes with V2G, charging and discharging in non-driving periods become much more frequent in order to profit from electricity arbitrage.
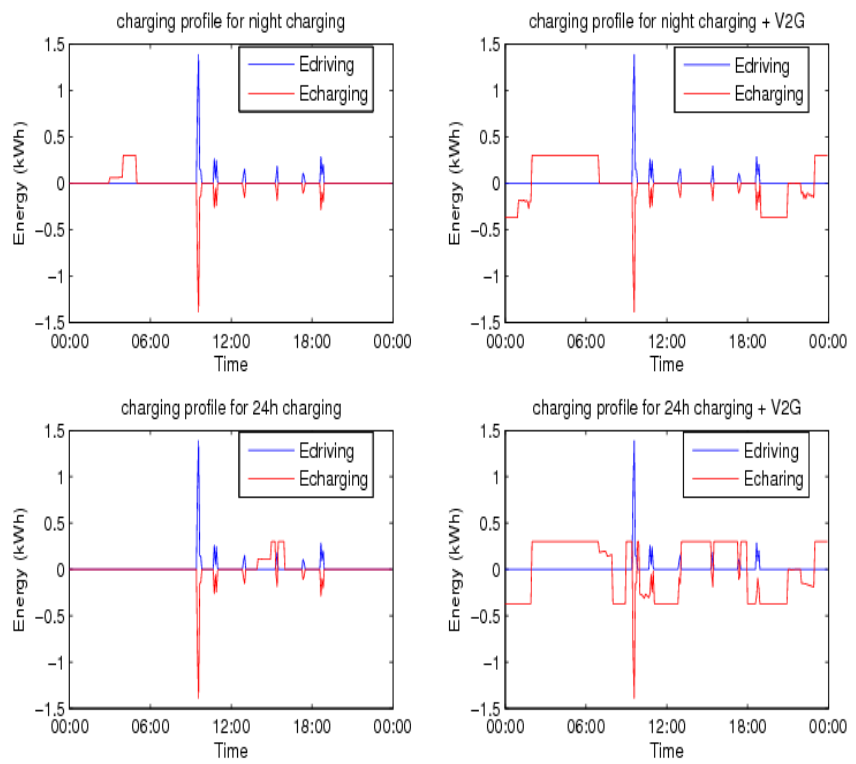

Fig. 4. Charging profiles with 5 minute resolution on March $2^{\text {nd }} 2003$

The corresponding SOC variations for different charging schemes are given in Fig. 5.
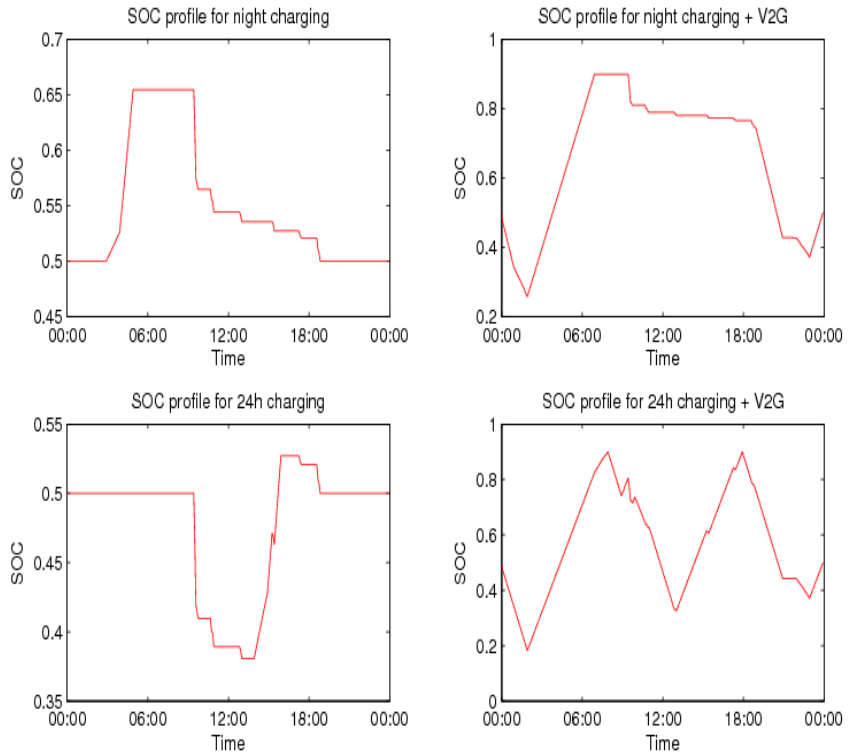

Fig. 5. SOC profiles with 5 minute resolution on March $2^{\text {nd }} 2003$

The resulting charging costs for different charging schemes on March $2^{\text {nd }} 2003$ are listed in Table II. For the intra-day case, the charging schemes with V2G options are obviously much more cost-effective than the other two due to the enriched flexibility; while the difference between the night charging and 24h charging is very small as the cheapest electricity price in the day time is very close to the cheapest electricity price at night.

TABLE II

CHARGING COST COMPARISON FOR MARCH 2ND 2003

\begin{tabular}{l|c}
\multicolumn{1}{c}{ Charging Options } & Charging Cost (DKK) \\
\hline night charging & 0.227 \\
\hline night charging + V2G & -3.1785 \\
\hline 24h charging & 0.1816 \\
\hline 24h charging + V2G & -6.3705 \\
\hline
\end{tabular}

\section{B. Monthly Performance}

By repeating the intra-day simulation, the charging profiles and the charging costs can be derived rather simply. As presented in Table III, the charging option "24h charging + V2G" is the least expensive, which costs approximately one third of the most expensive option "night charging". Again, there is little cost difference between "night charging" and " $24 \mathrm{~h}$ charging" due to the fact that cheap electricity periods are mostly in midnight.

TABLE III

CHARGING COST COMPARISON FOR MARCH 2003

\begin{tabular}{l|c}
\hline \multicolumn{1}{c|}{ Charging Options } & Charging Cost (DKK) \\
\hline night charging & 33.4762 \\
\hline night charging + V2G & 16.1366 \\
\hline 24h charging & 33.3772 \\
\hline 24h charging + V2G & 10.7202 \\
\hline
\end{tabular}


Among the various ways of assessing the lifetime consumption of a battery, the rainflow counting algorithm simply counts the number of cycles for each level of DOD in the operational period and assumes that the amplitude of DOD determines the fraction of the lifetime that is consumed [18]. In Figure 6, an example that illustrates how the algorithm of rainflow counting is applied is presented. The original signals, which have been randomly created, represent the observed change of SOC over a certain time period. During the counting process, if one could imagine that the original curve is rotated $90^{\circ}$ clockwise, the shape of the curve would be similar to a pagoda roof. If a raindrop is further assumed to start falling from each peak and valley of the roof, the half cycle path belonging to a specific raindrop could be obtained as indicated by the colored lines in the middle figure in Figure 6. For instance, the raindrop that follows the blue path has a length of $90 \%$, implying a half cycle of $90 \%$ DOD; while the green path indicates a half cycle of $60 \%$ DOD. In the histogram, the number of partial cycles with different values of DOD is counted into bins of equal width, with the bin width of $1 \%$ DOD being the granularity level considered in the counting process.
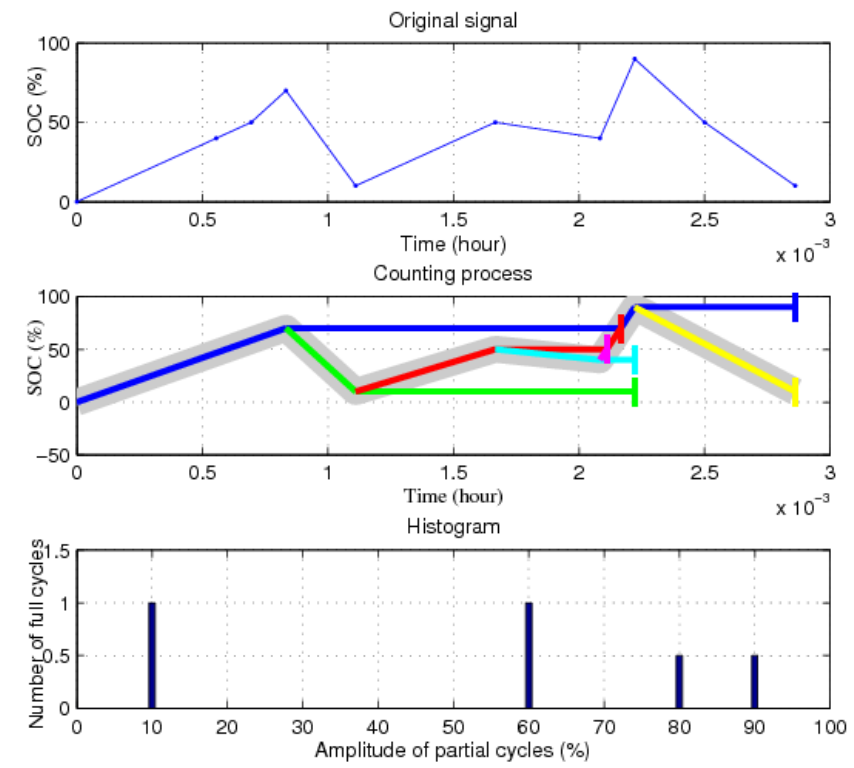

Figure 6: Illustration of the rainflow counting algorithm

By applying this post processing algorithm to the monthly charging profiles, while taking into account (3), the battery lifetime consumption for different charging schemes are calculated and illustrated in Fig. 7 and Table IV.

TABLE IV

LIFE TIME ESTIMATION FOR EV BATTERY

\begin{tabular}{l|c|c}
\hline \multicolumn{1}{c}{ Charging Options } & Life Usage (\%) & Expected Life (year) \\
\hline night charging & 0.5526 & 15.08 \\
\hline night charging + V2G & 0.6899 & 12.8 \\
\hline 24h charging & 0.5886 & 14.16 \\
\hline 24h charging + V2G & 0.9540 & 8.74 \\
\hline
\end{tabular}
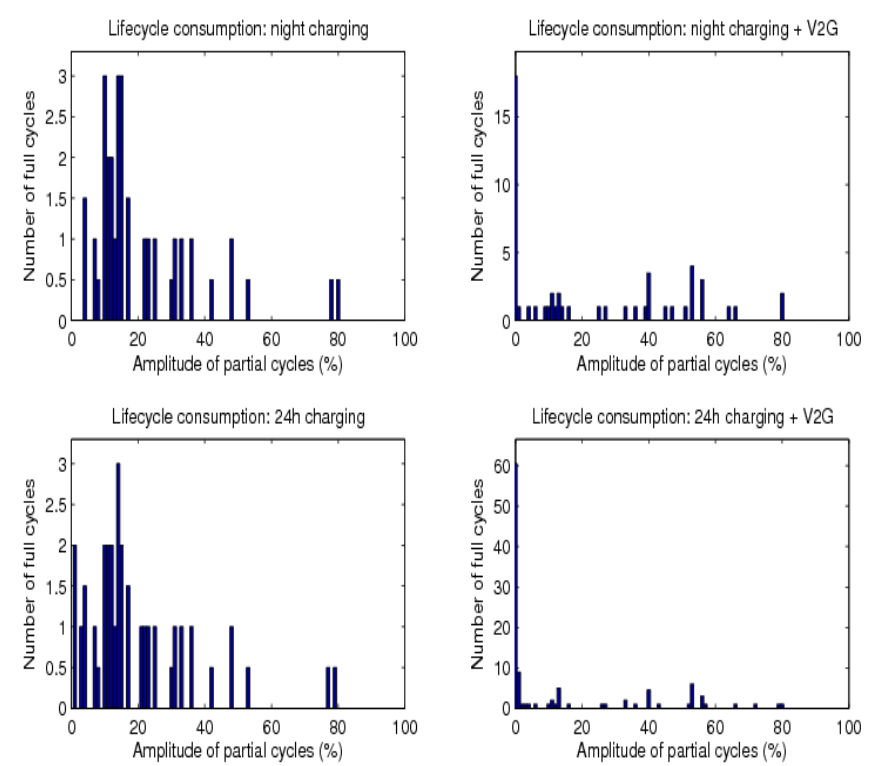

Fig. 7. Lifecycle consumption for the emulated EV battery in March 2003

As expected, the numbers of consumed partial cycles with small amplitudes are dramatically increased when the V2G options are included as illustrated in Fig.6. Although the charging option " 24 charging + V2G" is found to be the cheapest among the four schemes, the associated life usage for this scheme turns out to be the largest. $0.95 \%$ of the total battery lifetime has been consumed for the charging scheme “24 charging + V2G” in the studied period March 2003, and the expected battery life is therefore reduced by almost half compared to the night charging scheme.

\section{Annual Cost Comparison}

To provide an informative overall comparison, a simple approach is introduced by (4) to roughly estimate the annual cost $C_{a n n}$ for different charging schemes.

$$
C_{a n n}=\left(C_{c a p}+C_{c h a}\right) / L_{e x p}
$$

where $C_{c a p}$ and $C_{c h a}$ represents the capital cost of the battery and the charging cost incurred during the battery lifetime respectively, and $L_{\text {exp }}$ indicates the expected lifetime for different charging schemes. If the monthly charging cost for each scheme is assumed to be the same as the values presented in Table III, by further setting $C_{\text {cap }}$ to $120,000 \mathrm{DKK}$ which roughly represents the present market price of a $28 \mathrm{kWh}$ Lithium-ion battery, the annual cost for driving EV can be derived for each charging scheme. The result is summarized in Table V. Among the four options, the "night charging" scheme is found as the most cost-effective solution whereas the "24h charging + V2G" is found to be the most expensive solution due to its severe impact on battery service time and the high battery capital cost. 
TABLE V

ANNUAL COST COMPARISON FOR DIFFERENT CHARGING SCHEMES

\begin{tabular}{l|c}
\hline \multicolumn{1}{c|}{ Charging Options } & $C_{\text {ann }}(\mathrm{DKK} /$ year $)$ \\
\hline night charging & 8359 \\
\hline night charging + V2G & 9569 \\
\hline 24h charging & 8875 \\
\hline 24h charging + V2G & 13859 \\
\hline
\end{tabular}

\section{CONCLUSION}

This paper has provided a numerical comparison of four different optimal charging schemes, namely night charging, night charging with V2G, 24 hour charging and 24 hour charging with V2G, on the basis of real driving data and electricity price of Denmark in March 2003. Based on a best case scenario i.e. flawless forecasting, optimal charging schemes with 5 minute resolution are found by solving a mixed integer programming problem, and compared from both short-term and long-term perspective.

It has been found that the "night charging" scheme exhibits the lowest annual cost of using the EV. On the contrary, although the V2G option can to a great extent reduce the charging cost, its severe impact on the battery lifetime noticeably increases the annual cost of using the EV. This also implies the importance of judicious designs for V2G operations, which shall not only account for the value of V2G but also suppress its negative effects. Difference between "night charging" and "24h charging” appears to be small as the electricity price is normally cheap around midnight. This may indicate the need for establishing a residential charging infrastructure could come before the need for having a public charging infrastructure.

The EV economics are heavily dependent on the pattern of electricity prices and EV users as well as the other important factors, such as tax and subsidies. The study performed in this paper is therefore more informative than conclusive. To deliver an unbiased assessment of different charging schemes, collecting the sufficient representative information, refining the modeling assumptions, investigating the grid impacts and designing appropriate validation approaches are considered as future work. For the V2G possibilities, instead of providing bulk energy back to the grid as presented in this study, it would also be interesting to examine the economy of providing ancillary services to the power system operators with various risk-averse algorithms.

\section{REFERENCES}

[1] J. Lopes, F. Soares and P. Almeida, "Integration of electric vehicles in the electric power system," in Proc. of the IEEE, vol. 99, issue 1, pp. 168-83, Jan. 2011.

[2] T. Lan, J. Hu, G. Wu and S. You, "Optimal charge control of electric vehicles in electricity markets," presented at the $4^{\text {th }}$ Int. Workshop on Intelligent Information Management Systems and Technology, Shanghai, China, 2011

[3] J. Hu, S. You, and J. Østergaard, "Optimal charging schedule of an electric vehicle fleet," presented at the $46^{\text {th }}$ Int. Universities Power Engineering Conf., Soest, Germany, 2011.
[4] N. Rotering and M. Ilic, "Optimal charge control of plug-in hybrid electric vehicles in deregulated electricity markets," IEEE Trans. Power System, vol. 26, issue 3, pp. 1021-1029, Aug. 2011.

[5] T. K. Kristoffersen, K. Capion and P. Meibom, "Optimal charging of electric drive vehicles in a market environment,” Applied Energy, vol. 88, issue 5, pp. 1940-1948, Dec. 2010.

[6] W. Kempton and J. Tomic, "Vehicle-to-grid power fundamentals: Calculating capacity and net revenue," Journal of Power Sources, vol. 99, pp. 168-183, Dec. 2005.

[7] W. Kempton and J. Tomic, "Vehicle-to-grid power implementation: From stabilizing the grid to supporting large-scale renewable energy," Journal of Power Sources, vol. 144, issue 1, pp. 280-294, Jun. 2005.

[8] O. Sundstrom and C. Binding, "Optimization methods to plan the charging of electric vehicle fleets," in Proc. 2010 ACEEE Int. Conf. on Control, Communication, and Power Engineering, pp. 323-328

[9] J. A. p. Lopes, F. J. Soares and P. M. Almeida, "Smart charging strategies for electric vehicles: enhancing grid performance and maximizing the use of variable renewable energy resources," in Proc. 2009 EVS24 Int. Battery, Hybrid and Fuel Cell Electric VEHICLE Symposium

[10] K. Clement, E. Haesen and J. Driesen, "Coordinated charging of multiple plug-in hybrid electric vehicles in residential distribution grids," in Proc. 2009 IEEE Power Systems Conf. and Exposition, pp. 1-7

[11] Z. Ma, D. Callaway and I. Hiskens, "Decentralized charging control for large populations of plug-in electric vehicles,” in Proc. 2010 IEEE Conf. on Decision and Control, pp. 206-212

[12] A. Aabrandt, P.B. Andersen, A.B. Pedersen and S. You, "Prediction and optimization methods for electric vehicle charging schemes in the EDISON project,” to be presented at 2012 IEEE PES Conference on Innovative Smart Grid Technologies

[13] B. Lunz, H. Walz and D. U. Sauer, “Optimizing vehicle-to-grid charging strategies using genetic algorithms under the consideration of battery aging,” in Proc. 2011 IEEE Conf. on Vehicle Power and Propulsion Conference, pp. 1-7

[14] F. Marra, M. M. Jensen, "Power quality issues into a Danish LV grid with electric vehicles,” presented at IEEE EPQU, Lisbon, Portugal, 2011

[15] C. Søndergren, N. C. Bang, C. Hay, M. Togeby, and J. Østergaard, "Electric vehicles in future market models, Edison project," Danish Energy Association et al, DK, Tech. Rep. 2011.

[16] L. Christensen, "Edison project report: WP1.3 Electric vehicles and the customers,” DTU Transport, DK, Tech. Rep. 2011.

[17] www.axeonpower.com

[18] S. You and C. N. Rasmussen, "Generic modeling framework for economic analysis of battery systems," presented at the IET Renewable Power Generation Conf., Edinburgh, UK, 2011. 